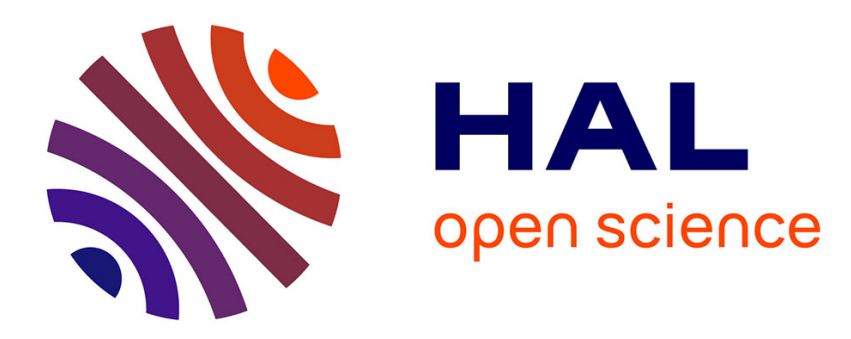

\title{
Built In-Stack Localisation Services with ALO
}

Adrien van den Bossche, Réjane Dalce, Thierry Val

\section{To cite this version:}

Adrien van den Bossche, Réjane Dalce, Thierry Val. Built In-Stack Localisation Services with ALO.

IFIP IEEE PEMWN 2020, Dec 2020, Berlin, Germany. hal-02984110

\section{HAL Id: hal-02984110 \\ https://hal.science/hal-02984110}

Submitted on 30 Oct 2020

HAL is a multi-disciplinary open access archive for the deposit and dissemination of scientific research documents, whether they are published or not. The documents may come from teaching and research institutions in France or abroad, or from public or private research centers.
L'archive ouverte pluridisciplinaire HAL, est destinée au dépôt et à la diffusion de documents scientifiques de niveau recherche, publiés ou non, émanant des établissements d'enseignement et de recherche français ou étrangers, des laboratoires publics ou privés. 


\title{
Built In-Stack Localisation Services with ALO
}

\author{
Adrien van den Bossche ${ }^{1}$, Réjane Dalcé ${ }^{2}$, Thierry Val ${ }^{1}$ \\ ${ }^{1}$ Institut de Recherche en Informatique de Toulouse, Université Toulouse 2 Jean Jaurès, France \\ ${ }^{2}$ Institut de Recherche en Informatique de Toulouse, Institut National Universitaire Champollion, France \\ $\{$ adrien.van-den-bossche, rejane.dalce, thierry.val\}@irit.fr
}

\begin{abstract}
Localisation is a major topic in the Wireless Sensor Networks and the Internet Of Things. This Work-in-Progress paper introduces ALO, an Ad-hoc Localisation Object proposed to help in designing in-stack localisation services, i.e. a service that enables the computation of mobile node positions, relying only on information from the protocol stack such as signal properties, RSSI, message timestamps, neighbour tables and routing tables. The paper presents ALO and then focuses on the implementation of a Time-Of-Flight ranging process using ALO. A suitable ranging protocol, minimizing overhead, is presented and evaluated on the LocURa4IoT testbed, using Ultra-Wide Band (UWB)-based wireless sensor nodes. The results show that the ranging accuracy is under $20 \mathrm{~cm}$ in indoor Line of Sight conditions, using this ranging protocol included in regular traffic messages, instead of using dedicated ranging messages.
\end{abstract}

Index Terms-Localisation Service; Ranging protocols; Time Of Flight; Testbed; Localisation \& Positionning

\section{INTRODUCTION}

Industrial indoor applications of localisation such as asset tracking involve adding a small electronic device to the parcel. These connected devices embark a finite amount of energy and communicate with each other using radio signals. While these devices may already collect environmental measurements (temperature, humidity level...), it would be cost effective to use them to generate localisation information. While the Global Navigation Satellite Systems (GNSS) like GPS, GLONASS or Galileo are now very popular, these technologies have two main drawbacks: first, because several Lineof-Sight links between the satellites and the device are required to compute the mobile position, the localisation service is usually unavailable in indoor environments such as warehouses or at home. Secondly, the GNSS receiver consumes a significant amount of energy, which is an important limitation for small, battery-powered embedded devices. The industry is thus looking for an alternative to these technologies, and the Wireless Sensor Networks (WSNs) are very likely to play a key role in the design of indoor Real Time Location Systems (RTLS). The advent of the Internet of Things (IoT), where all electronic devices can be connected to the Internet via short or long-range wireless links [1], only serves to hasten this development as many new applications require this feature.

So far, the research community has addressed this situation by proposing various protocols and algorithms suitable for various situations. This paper goes further by introducing the concept of In-Stack Localisation Services: this approach allows the existence of a localisation service which only relies on the data already present in the various layers of the protocol stack.
No dedicated localisation sensors or additional positioning systems like GNSS are necessary. We also introduce ALO, an Ad-hoc Localisation Object which supports the design of in-stack localisation services. In this paper, we focus on Timeof-Flight ranging process, which consists in generating transmission and reception timestamps via exchanged messages in order to compute the distance between wireless nodes.

The remainder of this paper is organised as follows: after a brief presentation of the objectives and the related work, we present the ALO model and a suitable ranging protocol implemented in ALO. Then, an experimentation on a real testbed is presented in order to analyse the performance of the ranging process.

\section{OBjectives}

The ultimate goal is to provide auto-localisation tools for the wireless nodes such as sensors or connected devices. The node's localisation is computed by the node itself (autolocalisation), only using the information generated by the communication system, without any localisation or positioningdedicated sensors such as accelerometers and GNSS receivers. To achieve this localisation, the wireless node relies on resources such as radio-signal properties (range-based localisation techniques) and neighbourhood-description tables implemented in the protocol stack (range-free localisation techniques). In this work, we plan to integrate the localisation task in the protocol stack: The final objective is to provide a Localisation Service through the protocol stack (figure 1) in the same way as the Data Service.

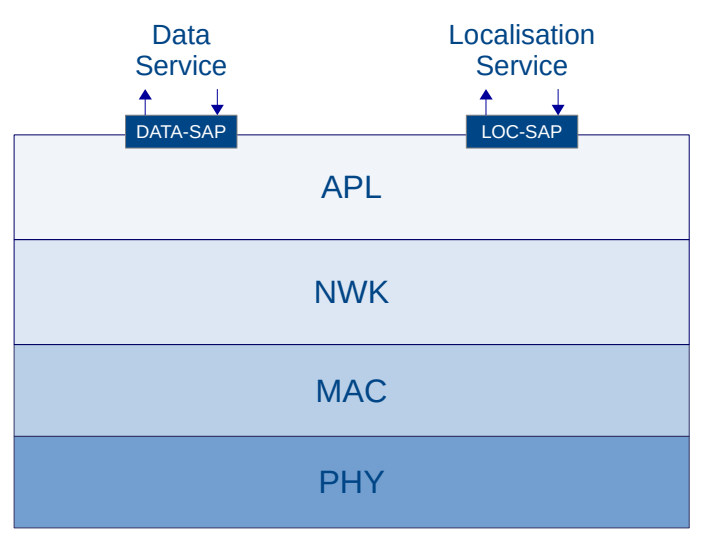

Fig. 1. The Localisation Service in the Protocol Stack 
The nodes may collaborate to achieve this localisation task, for example by using dedicated protocols to exchange anchor nodes' (i.e. fixed static nodes) positions or distances between nodes. Nevertheless, in the long run, the objective is to use an opportunistic ranging approach where the distance measurement messages are inserted in the data flow. This will contribute to minimising the protocol cost, both in terms of protocol overhead and energy consumption.

\section{RELATED WORK}

Submitting the term localisation to the IEEE research engine will return thousands of results. This body of work can be classified in various ways. Traditionally, localisation solutions have been seen as either range-based or range-free schemes [3] [8]. In the range-based approach, radio signal characteristics such as Received Signal Strength Indication (RSSI) or Time-of-Flight (ToF) are used to compute the mobile's position. The studies described in [9], [10] and [12] represent this family of solutions: in [9], the authors propose a hybrid 3D localisation system for WSN, using a GNSS localisation technology and RSSI-based ranging, where the nodes operate under heavily constrained energy and computing resources (CPU, memory). The performance evaluation, done through simulation, indicates a localisation accuracy of $15 \mathrm{~m}$ on an indoor environment. The study in [10] addresses the issue of localising a human being wearing a Bluetooth Low Energy (BLE) iBeacon. This ranging technique uses the RSSI to estimate the distance between two BLE devices. The ranging accuracy is about 1-meter in Line-Of-Sight (LOS) situations. As the best ranging precision results are generally obtained using Time-of-Flight [11], the work in [12] focuses on ToF over UWB.

On the other hand, the range-free approach exploits connectivity data organised in neighbour tables or distance-vector metrics [4]. One the most popular ranged-free localisation protocol is DV-hop [5] which is highly adapted to multi-hop mesh topologies. An improvement to DV-hop was proposed in [7] by selecting the three best anchors around the mobile node. The work in [6] introduced "Checkout DV-hop" which improves the localisation accuracy by $30 \%$ thanks to the introduction of a new step that consists in reusing the distance between the mobile and the nearest anchor, computed by DVHOP, to adjust the localisation result.

In all these approaches, the focus has been to improve accuracy. For example, in [12], several ranging protocols based on the IEEE 802.15.4-2011 Ultra-Wide Band Physical Layer are introduced. The ranging error is studied on a real testbed and a precision of $15 \mathrm{~cm}$ is obtained in a LOS context.

In order to support cooperative localisation, the localised nodes must be able to broadcast their position, allowing unlocalised nodes to learn the existence of new reference points. In [13], three protocols enabling localisation announcement are presented and compared. In the range-free context, [14] proposes a protocol to broadcast the node localisation information and proposes some trusted mechanisms.
Other solutions based on Angle Of Arrival, Phase of Arrival and other techniques could also be described here but the key takeaway is as follows: so far, most of the proposed schemes have focused on improving performance while losing sight of the reason why a network is deployed, which is to transport data. Unfortunately, most solutions consider the network as being dedicated to localisation with only one protocol being executed, the ranging protocol. Dual radio solutions such as [20] deploy two parallel networks in order to reap the benefits of existing and widely-disseminated technologies such as Bluetooth as well as the high precision provided by UWB. Separating the frequency bands used by the data and localisation services shields them from each other. In this paper, we introduce a model which will facilitate the coexistence of these services in the same radio band. The next section describes the model and introduces a suitable protocol for enabling in-stak localisation services.

\section{Building In-STACK LOCALiSATION SERVICES}

A. ALO: an Ad-hoc Localisation Object for in-stack localisation services

Our goal is to propose a Localisation Service built in the protocol stack, which operates in parallel of the Data Service (message transport service): each time a message is sent over the network, the Localisation Service in the receiver will attempt to localise the transmitting node in order to associate position-related metadata to the received message. The envisioned Localisation Service does not require any localisationdedicated sensor, subsystems such as a GNSS-receiver or inertial sensors. However, every piece of information available in the protocol stack can be used to determine the node localisation. This information may be available at various level of the protocol stack, such as the PHY-layer (signal properties, RSSI, ToF), the MAC-layer (neighbouring, connectivity), NWK-layer (routing table, distance-vector tables), APL-layer (known positions: anchors nodes and mobile nodes). Every protocol layer should share this information to compute the localisation data.

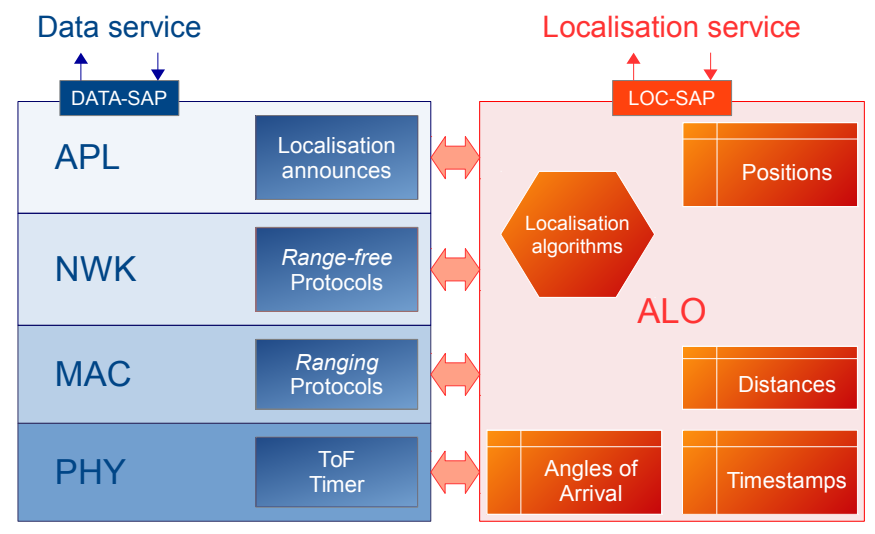

Fig. 2. The structure of ALO

On this basis, we propose ALO, an Ad-hoc Localisation Object placed alongside the protocol stack (figure 2). ALO: 
- has a direct access to each layer of the protocol stack. Thanks to this direct access, ALO can:

- get information from each layer, such as RSSI, message timestamping or local information stored in the tables (neighbour tables, routing tables...),

- trigger the execution of localisation or ranging protocols: timestamp exchanges (TWR), distance-vector broadcasts (DV-HOP), neighbour discovery and ranging (iBeacon) to compute new localisation information or confirm old positions. These protocols can request dedicated messages or be included in existing messages such as DATA, ACK or BEACON.

- memorises localisation information such as known positions, known distances between nodes (ranging results), message timestamps or Angle of Arrival (AoA),

- implements one or more localisation algorithms fed by all information available to ALO. Once a position has been computed, the result is timestamped and recorded in a local table. A trust metric should be associated to the position, such as a confidence or a dilution of precision (DOP), etc. If several localisation algorithms, ranging protocols or range-free protocols are available, a comparison between the various solutions can be done using ALO.

\section{B. 2M-TWR: a 2-Message Two-Way Ranging Protocol}

In this section, we present an improvement of the wellknown Two-Way Ranging (TWR) protocol (figure 3a). TWR is suitable for ALO, since it feeds the ALO's timestamp table and, thus, enables the distance computation (ranging). In addition, our improvement does not require any dedicated ranging messages and enables the collection of rangings using the background traffic. This improvement consists in the elimination of the REPLY message dedicated to carrying timestamps $t_{2}$ and $t_{3}$. If $t_{3}$ can be predicted with an acceptable precision, timestamps $t_{2}$ and $t_{3}$ can be carried by the ACK_REP message and the last message can be suppressed. This version of the TWR protocol is called two-message TWR (2M-TWR) (figure 3b).

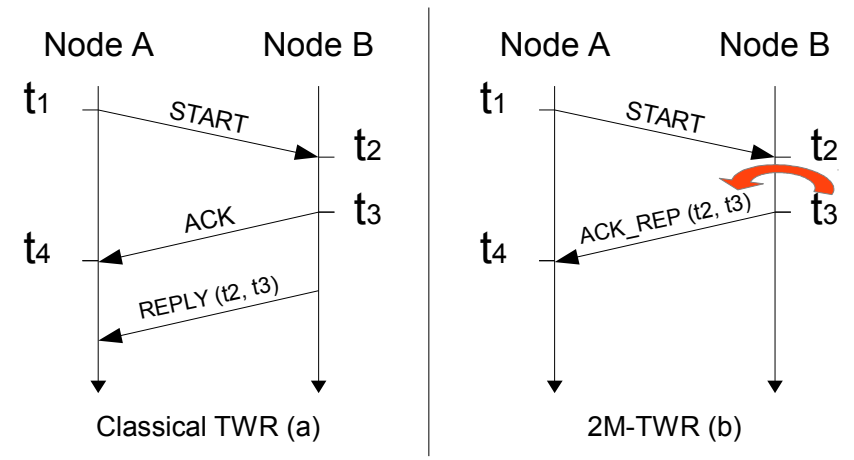

Fig. 3. TWR vs 2M-TWR protocol sequences

The ToF TWR formula remains unchanged (equation 1). Depending on the availability of High Precision Timers (HPT) at the Physical Level, 2M-TWR only requires the transmission of the timestamp difference $t_{3}-t_{2}$ on B's side. On the testbed implementation presented in section $\mathrm{V}$, the $t_{3}-t_{2}$ difference, the inter-frame delay, requires only 4 bytes, as will be justified in the next section.

$$
T_{o F}=\frac{t_{4}-t_{1}-\left(t_{3}-t_{2}\right)}{2}
$$

The ability to predict $t_{3}$ is very interesting: aside from reducing the number of messages, it also enables the ranging process between two nodes to fit in a standard DATA/ACK exchange. In other words, each time node A sends a DATA message to a 1-hop neighbour with an ACK requested, node A can also compute the distance to this neighbour and feed ALO's distance table.

TWR, SDS-TWR [19] and 2M-TWR protocols have been included in the ALO model (figure 2). While TWR and SDSTWR require respectively 3 dedicated messages (resp. 4 dedicated messages), 2M-TWR does not require any dedicated message and can be executed in an opportunistic fashion at each DATA/ACK exchange, feeding ALO's timestamp and distance tables. The symmetric nature of SDS-TWR is also a key difference to 2M-TWR: this symmetry allows for better mitigation of the influence of clock skew on the ranging results but typically imposes strong time constraints on the MAC layer.

A comparison of the ranging precision of these two protocols is presented in the next section. Thanks to 2M-TWR, the timestamps are collected with a minimal overhead, enabling distance computation to feed localisation algorithms.

\section{RESUlTS}

\section{A. Implementation details and testbed description}

ALO and 2M-TWR protocol have been implemented on the LocURa testbed [16] (fig. 4). This platform is based on the DecaWiNo node, running an IEEE 802.15.4-2011 UWB transceiver from DecaWave. The node MCU is a Freescale MK20DX256 (32 bit ARM Cortex-M4 $72 \mathrm{MHz}$ ) with 64kB RAM and $256 \mathrm{kB}$ program memory. The physical rates available are $110 \mathrm{kbps}, 850 \mathrm{kbps}$ and $6.8 \mathrm{Mbps}$.

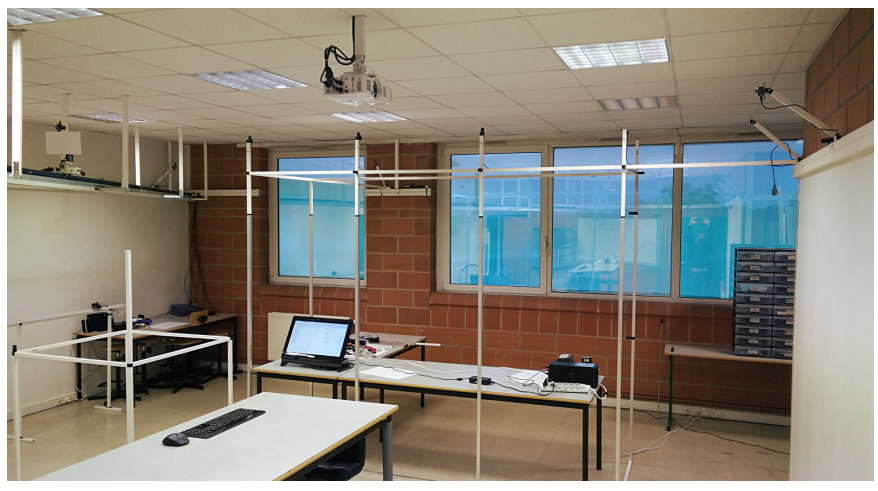

Fig. 4. The LocURa4IoT testbed 
The DecaWiNo (fig. 5) transceiver embarks a $64 \mathrm{GHz}$ timer for message timestamping purposes. Used in the context of ToF-based ranging, it enables $\mathrm{cm}$-level ranging precision. The $64 \mathrm{GHz}$ implies a 15.65 ps granularity, i.e. a $4.5 \mathrm{~mm}$ distance resolution. The timestamps are encoded on 3, 4 or 5 bytes, depending on the acceptable overflow period. For a fast protocol, i.e. a short inter-frame delay, the timestamps' size can be minimised as long as the HPT overflow period remains greater than the inter-frame delay $\left(t_{3}-t_{2}\right)$. The available sizes and corresponding overflow periods are indicated in table I. In the implementation used in this paper, the 2M-TWR protocol is running on the $6.8 \mathrm{Mbps}$ rate: the whole DATA/ACK is done in less than $3 \mathrm{~ms}$ with maximal PPDU frames (127 bytes). The timestamps are thus encoded on 4 bytes.

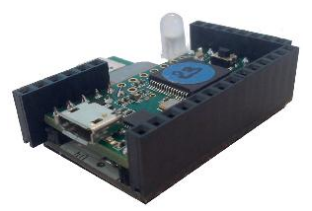

Fig. 5. The DecaWiNo sensor node

TABLE I

TIMESTAMP SIZE VS. OVERFLOW PERIOD

\begin{tabular}{|c|c|}
\hline Timestamp size (bytes) & Overflow Period (ms) \\
\hline 3 & 0.262 \\
\hline 4 & 67.1 \\
\hline 5 & 17179 \\
\hline
\end{tabular}

On the destination side, the DATA reception instant is timestamped $\left(t_{2}\right)$ by the transceiver. Then, the ACK transmission instant $\left(t_{3}\right)$ must be pre-configured and placed in the message. This operation can be done using the Delayed Transmission feature of DecaDuino [17]. The inter-frame delay is then computed by the receiver and added to the ACK in a dedicated field, as an unsigned 4-bytes integer. The 2M-TWR protocol overhead is thus limited to 4 bytes in the ACK message.

\section{B. Ranging Performance Evaluation}

In this section, we present the experimentation and the results obtained on the LocURa4IoT testbed. The experimentation setup is the following: two DecaWiNos implementing the ALO model and executing the three ranging protocols TWR, SDS-TWR and 2M-TWR are positioned in a LOS indoor environment. The first node is fixed (anchor) and the second is mounted on software-controlled tracks (mobile): the distance between the two nodes is variable and automatically set through the experiment scenario. The three protocols are executed in three different experiments, with 150 rangings for 10 distances from $0.5 \mathrm{~m}$ to $5 \mathrm{~m}$, for each protocol.

The obtained results indicate an average absolute ranging error less than $20 \mathrm{~cm}$ for each protocol. The reference protocols TWR and SDS-TWR obtain the smallest absolute ranging error with the smallest standard deviation: less than $18 \mathrm{~cm}$ with TWR (fig. 6) and less than $12 \mathrm{~cm}$ using SDS-TWR (fig. 7).
Concerning 2M-TWR (fig. 8) the standard deviation of the ranging error is greater, because of the Delayed Transmission feature. Nevertheless the ranging precision remains acceptable. In return, 2M-TWR outperforms TWR and SDS-TWR with a smaller protocol overhead and does not requires any dedicated message nor specific MAC constraints. 2M-TWR can be executed over standard network traffic while TWR or SDSTWR can be triggered at specific times to confirm a particular ranging, for example.

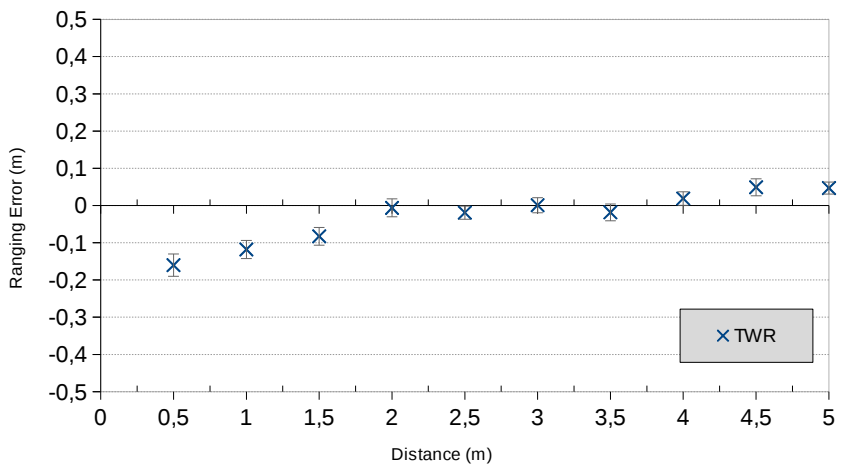

Fig. 6. Ranging error with the TWR protocol

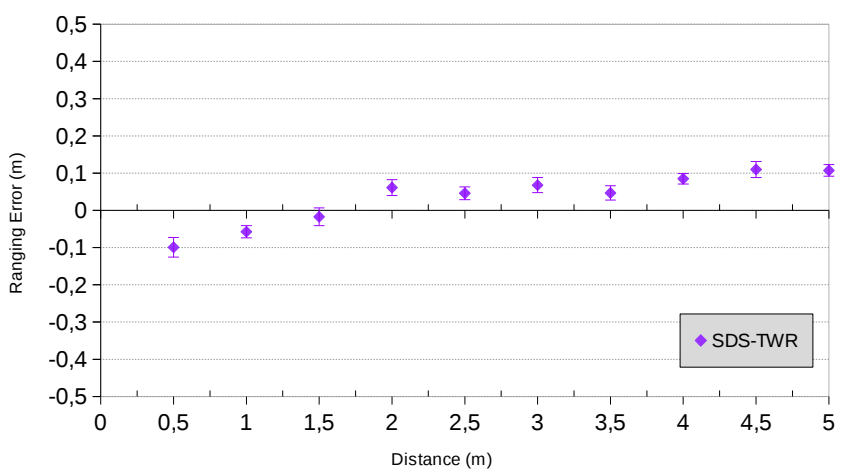

Fig. 7. Ranging error with the SDS-TWR protocol

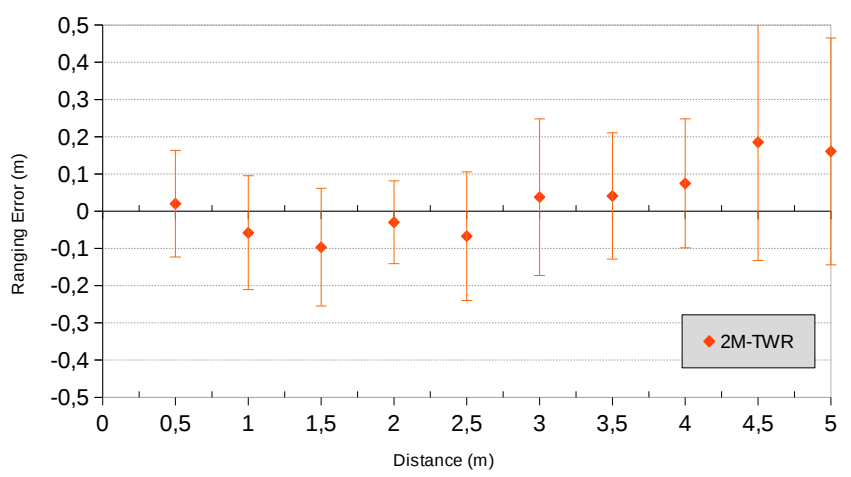

Fig. 8. Ranging error with the 2M-TWR protocol 


\section{CONCLUSION}

In this paper, we have introduced the concept of InStack Localisation Services which enables the provision of a localisation service only using information available in the protocol stack, without involving dedicated sensors or localisation systems like GNSS. We have introduced ALO, an Ad-hoc Localisation Object proposed to help in designing in-stack localisation services. In this cross-layer approach, ALO is given access to various pieces of information from all layers. Depending on the algorithm selected by ALO, a subset of the data will be used to compute the position. In this paper, we focused on Time-of-Flight ranging process, collecting transmission and reception timestamps to compute distances between the wireless nodes. A compliant ranging protocol, 2M-TWR, has been proposed and presented in the paper. This protocol enables the ranging process in every $\mathrm{DATA} / \mathrm{ACK}$, with a protocol cost of only 4-bytes per ACK message. A performance evaluation of the ranging error of 2M-TWR, compared to the well-known TWR and SDS-TWR protocols has been presented. The results show that the ranging precision is quite acceptable compared to the existing ranging protocols.

The next step of this Work-in-Progress is the implementation of a localisation algorithm in ALO to provide the entire Localisation Service. Mid-term perspectives concern the specification of ALO, with internal primitives and Service Access Point (SAP) specification, and the use of ALO in a cross-layer context, where the MAC and Network layers can feed ALO but also use localisation data to improve their own performance (spatial enhancement at MAC layer, geographical routing at NWK layer...). Non-Line of Sight (NLOS) conditions are also currently investigated in order to propose a confidence metric on each piece of information stored by ALO.

\section{REFERENCES}

[1] R. Khan, SU. Khan, R. Zaheer, S. Khan, Future Internet: The Internet of Things Architecture, Possible Applications and Key Challenges, in Frontiers of Information Technology (FIT), 2012 10th International Conference on, pp.257-260, 17-19 Dec. 2012

[2] Y. Ding, Y. Jin, L. Ren, K. Hao, An Intelligent Self-Organization Scheme for the Internet of Things, IEEE Computational Intelligence Magazine, 01/2013 pp 41-53.

[3] Y. Liu, Y. Zheng, Location, localization, and localizability: location awareness technology for wireless networks, Springer Science \& Business Media, 2010
[4] X.H. Ma, Z.G. Bing, Y.Q. Tang, Research on localization technology in wireless sensor networks, in Advanced research on computer education, simulation and modeling Springer, Berlin Heidelberg, 2011, pp. 392-398

[5] D. Niculescu, B. Nath, DV Based Positioning in Ad hoc Networks, Journal of Telecommunication Systems, 2003

[6] L. Gui, T. Val, A. Wei, R. Dalce, Improvement of Range-free Localization Technology by a Novel DV-hop Protocol in Wireless Sensor Networks, Ad Hoc Networks Journal, Elsevier, Vol. 24 N. Part B, jan 2015.

[7] L. Gui, T. Val, A. Wei. Improving Localization Accuracy Using Selective 3-Anchor DV-hop Algorithm, IEEE Vehicular Technology Conference (VTC 2011), San Francisco, 05/09/2011-08/09/2011

[8] R. Dalce, T. Val, A. Van den Bossche. Comparison of Indoor Localization Systems based on Wireless Communications, Wireless Engineering and Technology, Scientific Research Publishing, Irvine - USA, Vol. 2 No 4, october 2011.

[9] F. Chiti, R. Fantacci, S. Menci and A. Zappoli, Cooperative Localization Protocols for Wireless Sensor Networks, IEEE GLOBECOM 2007 IEEE Global Telecommunications Conference, Washington, DC, 2007, pp. 1048-1052

[10] A. Thaljaoui, T. Val, N. Nasri, D. Brulin. BLE Localization using RSSI Measurements and iRingLA. IEEE International Conference on Industrial Technology (ICIT 2015), Seville, 17/03/2015-19/03/2015, IEEExplore digital library, mars 2015.

[11] S. Lanzisera, D. T. Lin, K. S. J. Pister, RF Time of Flight Ranging for Wireless Sensor Network Localization, International Workshop on Intelligent Solutions in Embedded Systems, Vienna, 2006.

[12] F. Despaux, A. van den Bossche, K. Jaffrès-Runser, T. Val, N-TWR: An accurate time-of-flight-based $\mathrm{N}$-ary ranging protocol for Ultra-Wide band, Elsevier Ad Hoc Networks, Volume 79, 2018, Pages 1-19, ISSN 1570-8705,

[13] S. Tanvir, E. Schiller, B. Ponsard, A. Duda, Propagation Protocols for Network-Wide Localization Based on Two-Way Ranging, 2010 IEEE Wireless Communication and Networking Conference, Sydney, NSW, 2010, pp. 1-6

[14] A. M. Abbas, TrustLP: A trust-based localization protocol for wireless sensor networks, Modeling and Optimization in Mobile, Ad Hoc, and Wireless Networks (WiOpt), 2015 13th International Symposium on, Mumbai, 2015, pp. 529-536.

[15] A.S. Tonneau, N. Mitton, J. Vandaele, How to choose an experimentation platform for wireless sensor networks? Elsevier Adhoc networks, Elsevier, 2015, 30, pp.12.

[16] A. van den Bossche, R. Dalcé, N. Gonzalez, T. Val, LocURa: A New Localisation and UWB-Based Ranging Testbed for the Internet of Things, IEEE Indoor Positioning and Indoor Navigation conference, 24-27 september 2018, Nantes, France

[17] A. van den Bossche, R. Dalcé, N.I. Fofana, T. Val, DecaDuino: An Open Framework for Wireless Time-of-Flight Ranging Systems, IEEE/IFIP Wireless Days, WD 2016, Toulouse, 23-25/03/2016

[18] DecaWave Ltd. 2013, DWM1000 datasheet, last accessed on October 13,2020

[19] Y. Kim, W. Park, J. Kim and J. Kang, WLC18-2: Efficient LowComplexity Ranging Algorithm for Low Rate UWB Systems, IEEE Globecom 2006, San Francisco, CA, 2006, pp. 1-5.

[20] Marcin Kolakowski: Kalman filter based localization in hybrid BLEUWB positioning system, IEEE International Conference on RFID Technology and Application (RFID-TA) 2017 